\title{
Malmquist Histórico y de Pronóstico con Series de Tiempo como Modelo Temporal de Análisis Envolvente de Datos
}

\author{
Jhon J. Vargas ${ }^{(1)^{*}}$, Gerard Olivar ${ }^{(2)}$ y Edilberto Cepeda-Cuervo ${ }^{(3)}$ \\ (1) Fac. de Ingeniería, Universidad del Magdalena, cra 32 \#22-08 Sector San Pedro Alejandrino, Santa \\ Marta-Colombia. (e-mail: jvargass@unimagdalena.edu.co) \\ (2) Departamento de Matemáticas y Estadística, Universidad Nacional de Colombia, Campus la Nubia \\ Manizales-Colombia, (e-mail: golivart@unal.edu.co) \\ (3) Facultad de Ciencias, Departamento de Estadística, Universidad Nacional de Colombia, Edificio 405 \\ Bogotá-Colombia, (e-mail: ecepedac@unal.edu.co)
}

${ }^{*}$ Autor a quien debe ser dirigida la correspondencia

Recibido Sep. 21, 2015; Aceptado Nov. 23, 2015; Versión final Dic. 22, 2015, Publicado Jun. 2016

\section{Resumen}

Se presenta un nuevo modelo análisis envolvente de datos temporal que supera algunas debilidades de los modelos análisis de ventana e Índice Malmquist presentados en la literatura. Se construyen series temporales con datos de manufactura proporcionados por el Departamento Administrativo Nacional de Estadística. Para cada serie, se selecciona el mejor de un conjunto de modelos ARIMA y se establecen pronósticos para dos períodos. Se analizan cambios de eficiencia de los cinco centros industriales más grandes de Colombia. La aplicación del nuevo modelo muestra las ventajas que resultan al considerar más de dos períodos. Muestra también cómo se mejora el Malmquist clásico al cambiar variables univariadas determinísticas por series de tiempo. Basado en los resultados se concluye que el método está cerca de ser un análisis envolvente de datos en tiempo real. tiempo.

\section{Historical and forecast Malmquist with Time Series as a Temporal Data Envelopment Analysis Model}

\begin{abstract}
This paper presents a new temporal data envelopment analysis model that overcomes some weaknesses of the window analysis and Malmquist Index models reported in the literature. Time series are constructed with manufacturing data from the National Administrative Department of Statistics. For each series the best of a set of ARIMA models is selected, and a forecast for two periods is established. Changes in efficiency of the five largest industrial centers of Colombia are analyzed. For that, variables are located at different time positions including forecasts. The implementation of the new model shows the benefits of considering more than two periods. It also shows how classical Malmquist method is improved by changing deterministic univariate variables by time-series. Based on the results it is concluded that the method is close of being a data envelopment analysis in real time.
\end{abstract}

Keywords: data envelopment analysis, temporal model, Malmquist index, efficiency, time series. 


\section{INTRODUCCIÓN}

El Análisis Envolvente de Datos (AED ó DEA en inglés) es una técnica que ha sido ampliamente usada para evaluar el desempeño de diferentes sistemas organizacionales. AED es una técnica no paramétrica usada para evaluar las eficiencias relativas de un conjunto de Unidades de Decisión (UD ó DMU por sus siglas en inglés Decision Making Units) (Herrera et al., 2014). En la literatura se encuentran múltiples aplicaciones de los modelos AED, de las cuales cabe mencionar desempeños en el sector hospitalario (Varabyova y Schreyögg, 2013); plantas de tratamiento de aguas (Sala-Garrido y Hernández., 2012); políticas ambientales (Macpherson et al., 2013); industria petrolera (Al-Najjar y Al-Jaybajy 2012) y desempeño ambiental (Meng et al., 2013; Chang et al., 2013).

Una de las categorías en la que se clasifican los modelos AED es la de variación en los datos (Cook y Seiford, 2009). En esta categoría están las subcategorías "modelos basados en probabilidad" y "los datos en series de tiempo" ó "AED temporales". De éstos últimos se conocen dos en la literatura clásica: el análisis de ventana y el índice Malmquist. Hay evidencias que muestran que no se han registrado nuevos métodos AED temporales a parte de Análisis de ventana e Indice Malmquist (Toshiyuki et al., 2013; Sueyoshi y Goto, 2013). Una aproximación a Malmquist temporal con múltiples períodos ha sido estudiada en Sueyoshi y Goto (2013); en donde se calcula índice Malmquist en los años 2000 a 2009 para valoración ambiental de diferentes países de Europa, Asia y América. Sin embargo, en este trabajo no hay un índice Malmquist que reúna en una sola medida el desempeño histórico, ni considera técnicas de series de tiempo y tampoco hace combinaciones de todos las cambios temporales posibles de acuerdo al número de períodos, es decir, año 2000-2001, 2000-2002, ... 2000-2009, 2001-2002, ..., 2001-2009, ...,2008-2009. Esta sería una revisión interesante de cómo es el comportamiento del índice con estas combinaciones, y en consecuencia, en este artículo proponemos un nuevo método para cálculo de eficiencias temporales ofreciendo algunas ventajas sobre los dos AED temporales existentes.

La primera contribución AED temporal es el índice Malmquist en 1953 (Lin, 2015) y la segunda contribución es el análisis de ventana creado por G. Klopp en su tesis doctoral en 1985 (Cooper et al., 2007). La construcción de índice Malmquist bajo la metodología AED aparece con el Malmquist radial (Färe et al. 1992), también hubo esfuerzos por considerar un Malmquist no radial basado en holguras (Zhu, 1996; Tone, 2002; Chen 2003). Se han reportado mezclas entre los métodos análisis de ventana y Malmquist (Sueyoshi y Goto, 2001; Sueyoshi y Aoki, 2001), sin embargo, no se han evidenciado más avances en ampliación conceptual de este índice. Lo que sí se encuentra en la literatura moderna son aplicaciones de este AED temporal, como su uso en compañías petroleras (Sueyoshi y Goto, 2015), emisión de gas carbónico en compañías agrícolas de China (Boqiang y Rilong, 2015) e instituciones micro financieras de Kenya (Mahinda y Meoli, 2015). Este último artículo ha usado un procedimiento de bootstrap propuesto por Simar y Wilson (Simar y Wilson, 2007) para obtener intervalos de confianza en los índices Malmquist. Otras publicaciones se refieren al uso de Malmquist en el estudio de comercio de carbón, gas y aceite en 40 países (Sheng et al., 2015) y al estudio de puertos en México (Delfín y Navarro, 2015).

El Malmquist evalúa el efecto de eficiencia y el efecto de frontera que experimentan las UDs. Para determinar el efecto de eficiencia una medida llamada "Catch-up", que traduce recuperación, es calculada para verificar si hubo progreso o decrecimiento en la eficiencia relativa de un periodo 1 al 2 de una UD, para lo cual se calcula el cociente entre la eficiencia con respecto a la frontera del período 2 y la eficiencia con respecto a la frontera del período 1. Estas eficiencias son calculadas con un modelo AED determinístico y, por lo general, se usa un CCR (por las siglas de los autores Charnes, Cooper y Rhodes) (Charnes et al., 1978). Para determinar el efecto de frontera, una medida llamada "cambio de frontera" es calculada mediante la media geométrica entre el cambio de frontera para la UD observada en el periodo 1 y el cambio de frontera para la UD observada en el periodo 2. El índice Malmquist se define como en (1) (Cooper et al., 2007):

$M I=\left[\frac{\delta^{1}\left(\left(x_{0}, y_{0}\right)^{2}\right)}{\delta^{1}\left(\left(x_{0}, y_{0}\right)^{1}\right)} \times \frac{\delta^{2}\left(\left(x_{0}, y_{0}\right)^{2}\right)}{\delta^{2}\left(\left(x_{0}, y_{0}\right)^{1}\right)}\right]^{1 / 2}$

En la ecuación (1) $\delta^{1}$ se refiere a la frontera de eficiencia del primer período y $\delta^{2}$ la frontera de eficiencia del segundo período. La notación $\left(x_{0}, y_{0}\right)$ simboliza el vector de entradas y salidas de la UD observada (aquella a la que se le quiere calcular la eficiencia) y el superíndice de $\left(x_{0}, y_{0}\right)$ indica si el vector de entradas y salidas ha sido observado en el período 1 ó 2 . Cada $\delta^{s}\left(\left(x_{0}, y_{0}\right)^{t}\right)$ en (1) $(s=1,2 ; t=1,2)$ se calcula corriendo un modelo AED CCR determinístico orientado a las entradas ó a las salidas, según si las UD controlan las primeras o las segundas; los modelos AED orientados a las salidas y a las entradas se pueden consultar en Cooper et al. (2007). Si se está orientado a las salidas, el índice Malmquist se calcula corriendo el modelo matricial (2) cuatro veces donde $s=1,2$ y $t=1,2$. 
$\delta^{s}\left(\left(x_{0}, y_{0}\right)^{t}\right)=\min _{\theta, \lambda} \theta$

sujeto a las siguientes restricciones: $x_{0}^{t} \geq X^{s} \boldsymbol{\lambda},(1 / \theta) y_{0}^{t} \leq Y^{s} \boldsymbol{\lambda}, L \leq e \boldsymbol{\lambda} \leq U, \boldsymbol{\lambda} \geq \mathbf{0}$.

Si $(L, U)=(1,1)$ en el modelo (2), este se convierte en un BCC (Banker, Charnes y Cooper), lo que se traduce en cálculo de eficiencias de retorno a escala variable (Zhu, 2014), adecuado para comparar UDs de diferente tamaño. Si $(L, U)=(0, \infty)$, (2) se define un modelo CCR, lo que se traduce en cálculo de eficiencias con retorno a escala constante, adecuado para comparar UDs de igual tamaño. En este modelo el vector $\lambda=\left(\lambda_{1}, \lambda_{2}, \ldots \lambda_{N}\right)^{\prime}$, donde $N$ es el número de UDs, los $\lambda_{i}$ son las variables que ayudan a construir la envolvente para formar la frontera de eficiencia; el vector $e=(1,1, \ldots 1)$ de tamaño $1 \times N ; X$ es la matriz de entradas y $Y$ es la matriz de salidas. Tanto $X$ como $Y$ se disponen de manera que el número de filas sean el número de entradas y salidas respectivamente, y el número de columnas sean el número de UDs. El vector $x_{0}^{t}$ es el de las entradas de la UD observada en el período $t$ y $y_{0}^{t}$ el vector de salidas de la UD observada en el período $t$.

La segunda contribución al AED temporal es el análisis de ventana creado por G. Klopp en su tesis doctoral en 1985 (Cooper et al., 2007), trata cada UD como si fuera diferente en cada tiempo. Al tener $N$ periodos de tiempo y $n$ UDs, se consideran $n \times N$ de ellas. En el análisis de ventana se escoge una "longitud de ventana" $p$ y se evaluan $n \times p$ eficiencias para cada ventana, en vez de $n \times N$, donde el número de ventanas depende del lapso de tiempo considerado. De esta forma se puede visualizar si las eficiencias de determinada UD experimentan tendencias al deterioro o se estabiliza en cierto nivel de eficiencia. Luego se calcula la media y varianza de la eficiencia como medidas explicativas de las eficiencias de cada unidad.

Algunas debilidades pueden ser resaltadas en el Análisis de ventana y en el índice Malmquist: Están diseñados para periodos cortos de tiempo (en el caso del Malmquist solo maneja dos períodos como se ve en los modelos (1) y (2)); no están diseñadas para pronóstico; las variables de entrada y salida son univariadas determinísticas y no tienen en cuenta la estructura de correlación de las variables de entrada y salida, pues ningún AED temporal asume series de tiempo como variables de entrada y salida.

No hay una metodología en la literatura AED que calcule la eficiencia teniendo en cuenta la historia de las variables de entrada y salida, y aunque podría decirse que el análisis de ventana lo hace, éste tiene en cuenta la historia de las eficiencias calculadas, no de cada variable de entrada y salida. Tampoco hay un Malmquist que reúna, en una sola medida, todos los cambios posibles de eficiencias de un período a otro, teniendo en cuenta los períodos de las series de entrada y salida.

Dadas las anteriores debilidades, en el presente artículo se establece un nuevo modelo que considera variables de entrada y salida como series de tiempo en vez de univariadas determinísticas. La ventaja es que se pueden considerar series estacionarias y no estacionarias y podrían ser largas series de tiempo, aprovechando las ventajas de pronóstico que la teoría de series temporales ofrece. Para documentar el uso del nuevo modelo se ha aplicado al cálculo de eficiencias de los cinco centros industriales más grandes de Colombia, escogiendo unas variables de salida y entrada, cada una de ellas, con valores variables en el tiempo cuyos datos han sido encontrados en la encuesta anual de manufactura (EAM) proporcionados por el Departamento Administrativo Nacional de Estadística de Colombia (DANE). Se analiza el índice Malmquist considerando toda la historia de las series, asumiendo más de dos períodos y tomando valores no observados en las series, es decir pronósticos, gracias a la selección del mejor modelo ARIMA de cada serie de tiempo.

Los resultados muestran un AED temporal con mayores ventajas que los que ofrecen los de la literatura, el Malmquist y análisis de ventana, calculando una eficiencia temporal que recoge la historia de las UDs y potencializando el uso de eficiencias temporales llevándonos a estar cerca de un AED en tiempo real.

\section{METODOLOGIA}

Se han elegido como UDs los cinco centros industriales más grandes de Colombia, Antioquia, Atlántico, Bogotá, Cundinamarca (sin Bogotá) y Valle, para los cuales se calculan los cambios de eficiencia aplicando el método propuesto. Se han definido para cada UD tres variables de salida y cinco variables de entrada, tales variables son series de tiempo cuya construcción se hizo extrayendo datos de la EAM del DANE Colombia entre los años 1992 hasta el 2010 (19 períodos). Como variables de salida se definieron las siguientes: 1) personal remunerado permanente (PRP), 2) producción bruta (PB) y 3) valor agregado (VA). Como variables de entrada se definieron las siguientes: 1) Consumo de energía eléctrica en $\mathrm{KW} / \mathrm{h}$, 2) total activos, 3) consumo intermedio, 4) prestaciones sociales y 5) sueldos y salarios. Detalles del significado de las variables se encuentran en Maldonado et al. (2009). 
En la figura 1 se muestra 5 de las 40 series de tiempo analizadas, estas cinco series corresponden al consumo de energía eléctrica en millones $\mathrm{KWH}$. Para facilidad en los cálculos todos los valores de las variables se han dividido por un millón, excepto PRP que se dividió por 1000.

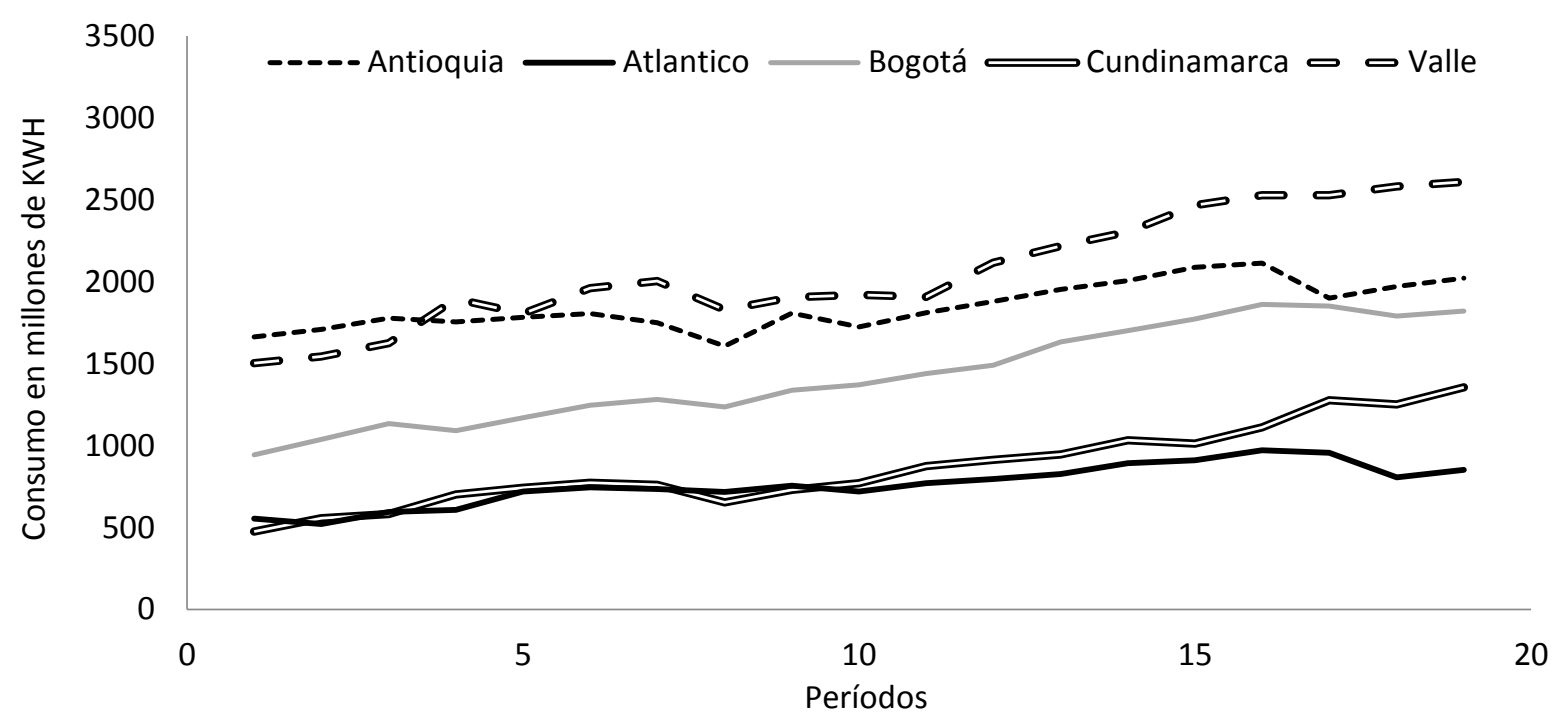

Fig. 1: Series de tiempo de consumo en $\mathrm{KWH}$ de 5 centros industriales

En el método propuesto una nueva medida se ha definido como Malmquist histórico, denotado por $M h$, el cual se define como la media geométrica de todos los índices Malmquist posibles de acuerdo con el número $n$ de períodos de tiempo que conforman las series tal como se aprecia en ecuación (3):

$M h=\sqrt[c]{M_{1,2} M_{1,3} \ldots M_{1, n} M_{2,3} M_{2,4} \ldots M_{2, n} \ldots M_{n-2, n-1} M_{n-1, n}}$,

donde $M_{i, j}$ es el índice Malmquist clásico calculado en los períodos $i$ y $j, i=1, \ldots, n-1 ; j=2, \ldots, n ; c$ es el número de combinaciones de los períodos dado por $c=\left(\begin{array}{l}n \\ 2\end{array}\right)$. Como supuesto primordial se establece que el nuevo AED temporal necesita que todas las series de tiempo de entradas y salidas tengan el mismo número de períodos. En el nuevo $M h$ se calculan los $M_{i, j}$, cada uno de los cuales se corre con el modelo (2), pero ahora $t=1, \ldots, n$ y $s=1, \ldots, n$. En (3), para el cálculo de $M h$, se ha elegido la media geométrica, ya que ésta es adecuada para calcular promedios cuando las cantidades son índices.

Definida la ecuación (3), se procedió a realizar el cálculo de todos los $M_{i, j}$ y por tanto el $M h$ para los períodos observados, haciendo $n=19$. Cada $M_{i, j}$ require de cuatro corridas del modelo (2), las cuales se realizaron mediante un programa escrito en Matlab. También se calculó $M h$ haciendo $n=21$, es decir, con períodos aún no observados 20 y 21, para lo cual se establecieron pronósticos haciendo uso del siguiente conjunto de modelos ARIMA (López et al., 2015): $\operatorname{ARIMA}(1,1,0), \operatorname{ARIMA}(2,1,0), \operatorname{ARIMA}(1,2,0)$, $\operatorname{ARIMA}(2,2,0), \operatorname{ARIMA}(0,0,1), \operatorname{ARIMA}(0,1,1), \operatorname{ARIMA}(0,1,2), \operatorname{ARIMA}(0,2,1)$ y $\operatorname{ARIMA}(0,2,2)$. Cada una de las 40 series de tiempo (8 variables y 5 UDs) fue modelada mediante todos los ARIMA mencionados y, para el pronóstico, en cada serie se escogió aquel modelo que producía el menor error cuadrático.

\section{RESULTADOS}

La Tabla 1 muestra las estimaciones de los modelos ARIMA para la serie de tiempo Antioquia en la variable PRP. En esta Tabla, se nota que modelo que mejor ajusta esta serie es el ARIMA $(0,1,2)$, con menor error cuadrático y con menor criterio de información Bayesiano (BIC por sus siglas en inglés). Estos cálculos también se hicieron para las otras 2 variables de salida y cinco de entrada así como para los otros cuatro centros industriales o UDs, para un total de 40 tablas como la Tabla 1.

La Tabla 2 muestra el pronóstico para la serie Antioquia en la variable PRP y los intervalos de confianza del $95 \%$, igual procedimiento se hizo para las otras variables y demás departamentos, para un total de 40 tablas como la Tabla 2. Cada uno de estos pronósticos es usado en ecuaciones (2) y (3), cuyos resultados permiten obtener el índice Malmquist histórico con pronóstico, es decir, teniendo en cuenta los períodos observados y los aún no observados 20 y 21. 
Tabla 1: Modelos ARIMA para serie Antioquia variable PRP

\begin{tabular}{|c|c|c|c|c|c|}
\hline & MS & \multicolumn{2}{|c|}{ coeficientes } & p valor & BIC \\
\hline ARIMA(1,1,0) & 17.928 & AR 1 & 0.651 & 0.005 & 3.024 \\
\hline & & Constante & -0.293 & 0.777 & \\
\hline ARIMA(2,1,0) & 17.159 & AR 1 & 0.410 & 0.114 & 3.071 \\
\hline & & AR 2 & 0.403 & 0.113 & \\
\hline & & Constante & 0.045 & 0.968 & \\
\hline ARIMA(1,2,0) & 17.770 & AR 1 & -0.496 & 0.041 & 2.951 \\
\hline & & Constante & $-0,160$ & 0.878 & \\
\hline ARIMA(2,2,0) & 15.778 & AR 1 & -0.339 & 0.19 & 2.918 \\
\hline & & AR 2 & 0.433 & 0.095 & \\
\hline & & Constante & -0.040 & 0.967 & \\
\hline ARIMA(0,0,1) & 134.09 & MA 1 & -0.947 & 0.000 & 5.097 \\
\hline & & Constante & 98.070 & 0.000 & \\
\hline ARIMA(0,1,1) & 22.830 & MA 1 & -0.333 & 0.175 & 3.266 \\
\hline & & Constante & -1.761 & 0.257 & \\
\hline ARIMA(0,1,2) & 11.475 & MA 1 & -0.800 & 0 & 2.669 \\
\hline & & MA 2 & -0.972 & 0 & \\
\hline & & Constante & -2.177 & 0.252 & \\
\hline ARIMA(0,2,1) & 20.322 & MA 1 & 0.287 & 0.265 & 3.085 \\
\hline & & Constante & -0.150 & 0.85 & \\
\hline ARIMA(0,2,2) & 18.723 & MA 1 & 0.308 & 0.247 & 3.089 \\
\hline & & MA 2 & -0.337 & 0.208 & \\
\hline & & Constante & -0.170 & 0.878 & \\
\hline
\end{tabular}

La Tabla 3 muestra las estimaciones de los parámetros de los modelos ARIMA seleccionados con menor error cuadrático, correspondientes al departamento de Antioquia para las tres variables de salida y cinco de entrada. Con estos modelos y los parámetros estimados se realiza el pronóstico como los de la Tabla 2. Igual procedimiento se hizo para los demás departamentos y la ciudad de Bogotá. Con estas estimaciones se tienen pronósticos de buena calidad para cada una de las variables temporales de entrada y salida de cada departamento (UD). El uso de tales pronósticos en ecuaciones (2) y (3) permite pronosticar lo siguiente: Cuáles pueden ser los desempeños históricos de las UD teniendo en cuenta los períodos observados y pronosticados; los cambios de eficiencia de todas las UD en los siguientes dos períodos aun no observados; las eficiencias instantáneas en los períodos, aun no observados, 20 y 21. Tales pronósticos ayudan a cada UD a corregir posibles fallas en los niveles de entradas y salidas.

Tabla 2: Pronóstico para serie Antioquia variable PRP

\begin{tabular}{|c|c|c|c|}
\hline Período & Pronóstico & Límite inferior & Límite superior \\
\hline 20 & 88.566 & 81.925 & 95.206 \\
\hline 21 & 88.123 & 74.447 & 101.800 \\
\hline
\end{tabular}

Tabla 3: Estimación de parámetros modelo ARIMA para Antioquia en 5 variables

\begin{tabular}{|c|l|c|c|c|c|c|c|}
\hline & \multicolumn{1}{|c|}{ Variable } & Modelo & coeficiente & p-valor & Constante & p-valor \\
\hline \multirow{5}{*}{ variable salida } & \multirow{2}{*}{ PRP } & ARIMA(0,1,2) & MA 1 & -0.800 & 0.000 & -2.177 & 0.252 \\
\cline { 2 - 7 } & & & MA 2 & -0.972 & 0.000 & & \\
\cline { 2 - 7 } & PB & ARIMA $(0,2,1)$ & MA 1 & 0.893 & 0.006 & 61.650 & 0.276 \\
\cline { 2 - 7 } & VA & ARIMA $(1,2,0)$ & AR 1 & -0.514 & 0.035 & -2.500 & 0.980 \\
\hline \multirow{5}{*}{ variable entrada } & Consumo KWH & ARIMA $(0,2,1)$ & MA 1 & 0.949 & 0.004 & 0.710 & 0.874 \\
\cline { 2 - 8 } & Total activos & ARIMA $(0,1,1)$ & MA 1 & 0.892 & 0.000 & 857.660 & 0.000 \\
\cline { 2 - 8 } & Consumo intermedio & ARIMA $(0,2,1)$ & MA 1 & 0.891 & 0.005 & 52.050 & 0.222 \\
\cline { 2 - 8 } & Prestaciones sociales & ARIMA $(0,2,1)$ & MA 1 & 1.120 & 0.000 & -0.894 & 0.193 \\
\cline { 2 - 8 } & Sueldos y salarios & ARIMA $(0,2,1)$ & MA 1 & 0.9569 & 0.000 & 0.812 & 0.538 \\
\hline
\end{tabular}

La figura 2 muestra el cálculo de los Malmquist $M_{i, j}$, con $i=1, \ldots, 18$ y $j=2, \ldots, 19$; donde $i=1$ corresponde al año 1992 y $j=19$ corresponde al año 2010. En total, en esta figura, hay $c=\left(\begin{array}{c}19 \\ 2\end{array}\right)=171$ índices, es decir, los Malmquist calculados con todas las combinaciones posibles de los períodos $(1-2,1-3, \ldots, 1-19,2-3, \ldots, 2-$ $19, \ldots, 10-11, \ldots, 10-19, \ldots 18-19)$. El eje horizontal en la figura 2 corresponde al número de combinaciones de los períodos de dos en dos para el cálculo del índice Malmquist. Para facilitar la comprensión de esta figura, en la Tabla 4 se presentan las equivalencias entre el número de la combinación y los dos períodos 
combinados para calcular el índice. Por ejemplo la combinación 66 equivale a tener en cuenta los períodos 4 y 19, en las ocho series de tiempo correspondientes, para el cálculo del índice Malmquist (índice Malmquist entre el año 1995 al año 2010).

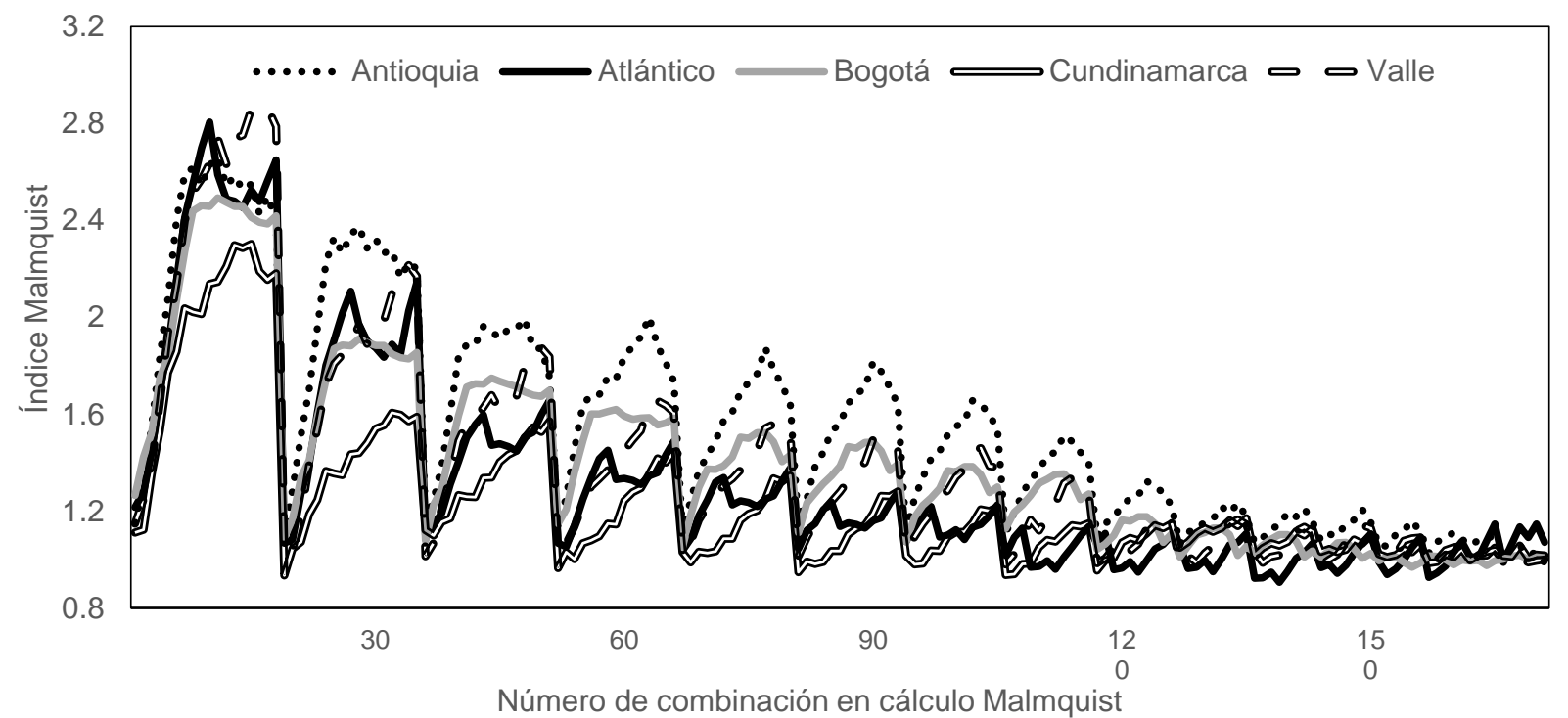

Fig. 2: Índices Malmquist para las combinaciones de períodos.

Tabla 4: Equivalencias entre el número de combinación y los dos períodos implicados

\begin{tabular}{|l|c|c|c|c|c|c|c|c|c|c|c|c|}
\hline № de combinación & 1 & 2 & $\ldots$ & 18 & 19 & 20 & $\ldots$ & 35 & 36 & $\ldots$ & 51 & 52 \\
\hline Períodos & $1-2$ & $1-3$ & $\ldots$ & $1-19$ & $2-3$ & $2-4$ & $\ldots$ & $2-19$ & $3-4$ & $\ldots$ & $3-19$ & $4-5$ \\
\hline № de combinación & $\ldots$ & 66 & 67 & $\ldots$ & 80 & 81 & $\ldots$ & 93 & 94 & $\ldots$ & 105 & 106 \\
\hline Períodos & $\ldots$ & $4-19$ & $5-6$ & $\ldots$ & $5-19$ & $6-7$ & $\ldots$ & $6-19$ & $7-8$ & $\ldots$ & $7-19$ & $8-9$ \\
\hline № de combinación & $\ldots$ & 116 & 117 & $\ldots$ & 126 & 127 & $\ldots$ & 135 & 136 & $\ldots$ & 143 & 144 \\
\hline Períodos & $\ldots$ & $8-19$ & $9-10$ & $\ldots$ & $9-19$ & $10-11$ & $\ldots$ & $10-19$ & $11-12$ & $\ldots$ & $11-19$ & $12-13$ \\
\hline № de combinación & $\ldots$ & 150 & 151 & $\ldots$ & 156 & 157 & 158 & $\ldots$ & 161 & 162 & $\ldots$ & 165 \\
\hline Períodos & $\ldots$ & $12-19$ & $13-14$ & $\ldots$ & $13-19$ & $14-15$ & $14-16$ & $\ldots$ & $14-19$ & $15-16$ & $\ldots$ & $15-19$ \\
\hline № de combinación & 166 & 167 & 168 & 169 & 170 & 171 & & & & & & \\
\hline Períodos & $16-17$ & $16-18$ & $16-19$ & $17-18$ & $17-19$ & $18-19$ & & & & & & \\
\hline
\end{tabular}

Nótese la importancia de considerar un Malmquist histórico sobre el Malmquist clásico. Por ejemplo, en la figura 2 si consideramos los períodos de tiempo 1-11 (número de combinación 10 en eje horizontal de esta figura), es claro que Atlántico muestra mayor cambio de eficiencia $\left(M_{1,11}\right)$ que Cundinamarca y Antioquia. Sin embargo, en esta figura, se aprecia que si se calculara Malmquist con los períodos 7-8 (número de combinación 94) o con los períodos 2-19 (combinación 35), Atlántico comienza a mostrar menor índice Malmquist que Antioquia y, si se avanza en la figura 2 hacia la derecha se nota que Atlántico decae cada vez más. Por ejemplo, obsérvese 6-19 (combinación 93) ó 8-19 (combinación 116), se observa un protagonismo de índice Malmquist en Antioquia.

Lo anterior ilustra la desventaja de considerar el índice Malmquist en solo 2 períodos de tiempo cuando las UDs poseen datos históricos y se desea obtener una eficiencia histórica; así que si de eficiencia histórica se trata, el Malmquist es completamente débil porque muestra cambios de eficiencia solo en dos períodos y no hay una medida que muestre cambios de eficiencia en más de dos períodos, ni que tenga en cuenta todos los cambios posibles de períodos, por lo tanto resulta una medida injusta para una UD como Antioquia si se considerara solo dos períodos específicos, por ejemplo 1-10, cuando es evidente en la figura 2 que Antioquia se destaca en su historia de eficiencia. Por ello, el Malmquist histórico presentado en este artículo posee mayor información que explica el índice Malmquist en toda la historia de las UDs. Las ventajas del Malmquist histórico sobre el tradicional básicamente son dos. La primera es que está capacitado para dar en una sola medida los cambios de eficiencia teniendo en cuenta todas las combinaciones posibles de períodos y por ello se ha denominado Malmquist histórico. La segunda ventaja es que puede calcular cambios de eficiencias de pronóstico por el hecho de manejar series de tiempo como variables de entrada y salida. 
Para que haya un mejor cálculo de Malmquist histórico hemos creado $M h$ que reúne todos los cambios posibles de eficiencias en todos los períodos. En la Tabla 5 se muestran los índices Malmquist históricos calculados mediante ecuación (3) y clasificados de mayor a menor. En esta Tabla también se muestran los Mh con pronóstico en los períodos 20 y 21.

Tabla 5: Índices $M h$ para 5 centros industriales

\begin{tabular}{|l|c|c|}
\hline & Índice Malmquist histórico $M h$ & $\begin{array}{c}\text { Índice Malmquist } M h \\
\text { con pronóstico incluyendo períodos 20 y 21 }\end{array}$ \\
\hline Antioquia & 1.500 & 1.473 \\
\hline Bogotá & 1.360 & 1.338 \\
\hline Valle & 1.340 & 1.336 \\
\hline Atlántico & 1.268 & 1.270 \\
\hline Cundinamarca & 1.203 & 1.209 \\
\hline
\end{tabular}

La Tabla 5 muestra eficiencias temporales obtenidas con pronóstico, lo cual puede ser muy útil en las organizaciones. Nótese en la Tabla 5 que el índice temporal favorece al departamento de Antioquia y a la ciudad de Bogotá, tal como se observa en la figura 2. La Tabla 6 muestra cuáles pueden ser los índices Malmquist esperados para el cambio de los períodos aun no observados 20 y 21 . En la parte derecha de la Tabla 6 están las clasificaciones ordenadas de mayor a menor (lo que en la literatura DEA en inglés se conoce como ranking), se observa que Valle podría experimentar un ascenso en su participación mientras que Bogotá podría experimentar un descenso. Este análisis sería de gran utilidad para los desempeños en las organizaciones ya que permite, mediante las técnicas de pronóstico del presente artículo, anticiparse a los resultados según la tendencia histórica de las series de datos de entrada y salida de todas la UDs y corregir o diseñar estrategias para mejorar. La diferencia entre los resultados de la Tabla 5 y Tabla 6 es que, en la Tabla 5 se han usado todas las combinaciones posibles de períodos y en la Tabla 6 sólo dos períodos, el 20 y 21.

Tabla 6: Malmquist esperados según pronóstico períodos 20 y 21

\begin{tabular}{|c|c|c|c|}
\hline UD & $\begin{array}{c}\text { Pronóstico } \\
\text { Malmquist 20-21 }\end{array}$ & UD & $\begin{array}{c}\text { Pronóstico } \\
\text { Malmquist 20-21 }\end{array}$ \\
\hline Antioquia & 1.007 & Antioquia & 1.007 \\
\hline Atlántico & 1.006 & Atlántico & 1.006 \\
\hline Bogotá & 0.914 & Valle & 1.005 \\
\hline Cundinamarca & 0.997 & Cundinamarca & 0.997 \\
\hline Valle & 1.005 & Bogotá & 0.914 \\
\hline
\end{tabular}

Los pronósticos en el Malmquist también ayudan a establecer la eficiencia instantánea, por ejemplo, en la Tabla 7 se pueden observar las eficiencias de los períodos 20 y 21 bajo un AED CCR determinístico orientado a las salidas con retorno a escala constante (CCR-CRS output oriented, así se denota en la literatura clásica en inglés). Se puede esperar una buena participación en la eficiencia del departamento del Valle en los períodos instantáneos aun no observados 20 y 21, así que una buena recomendación para Valle es que si desea obtener el primer puesto en la clasificación AED, debe procurar aumentar los niveles de salida y disminuir los niveles de entradas en lo posible. De no hacerlo, y presentarse aumentos inesperados en entradas y disminuciones inesperadas en salidas, podría no alcanzar tal clasificación en los períodos específicos instantáneos 20 y 21.

Tabla 7: Eficiencias pronosticadas para períodos 20 y 21

\begin{tabular}{|c|c|c|}
\hline & $\begin{array}{l}\text { Eficiencias pronosticadas para período } \\
20\end{array}$ & $\begin{array}{l}\text { Eficiencias pronosticadas para período } \\
21\end{array}$ \\
\hline Antioquia & 1 & 1 \\
\hline Atlántico & 1 & 1 \\
\hline Bogotá & 1 & 1 \\
\hline Cundinamarca & 1 & 1 \\
\hline Valle & 1.054 & 1.052 \\
\hline
\end{tabular}


Una de las grandes ventajas que ofrece el índice Malmquist es que se puede separar el efecto de frontera y el efecto de recuperación (frontier shift y catch-up en los términos originales). En este análisis se han separado estos dos efectos para todas las combinaciones posibles de períodos. En la figura 3 se muestra el efecto de frontera comparado con el índice Malmquist que han resultado de una gran similitud con ligeras diferencias.
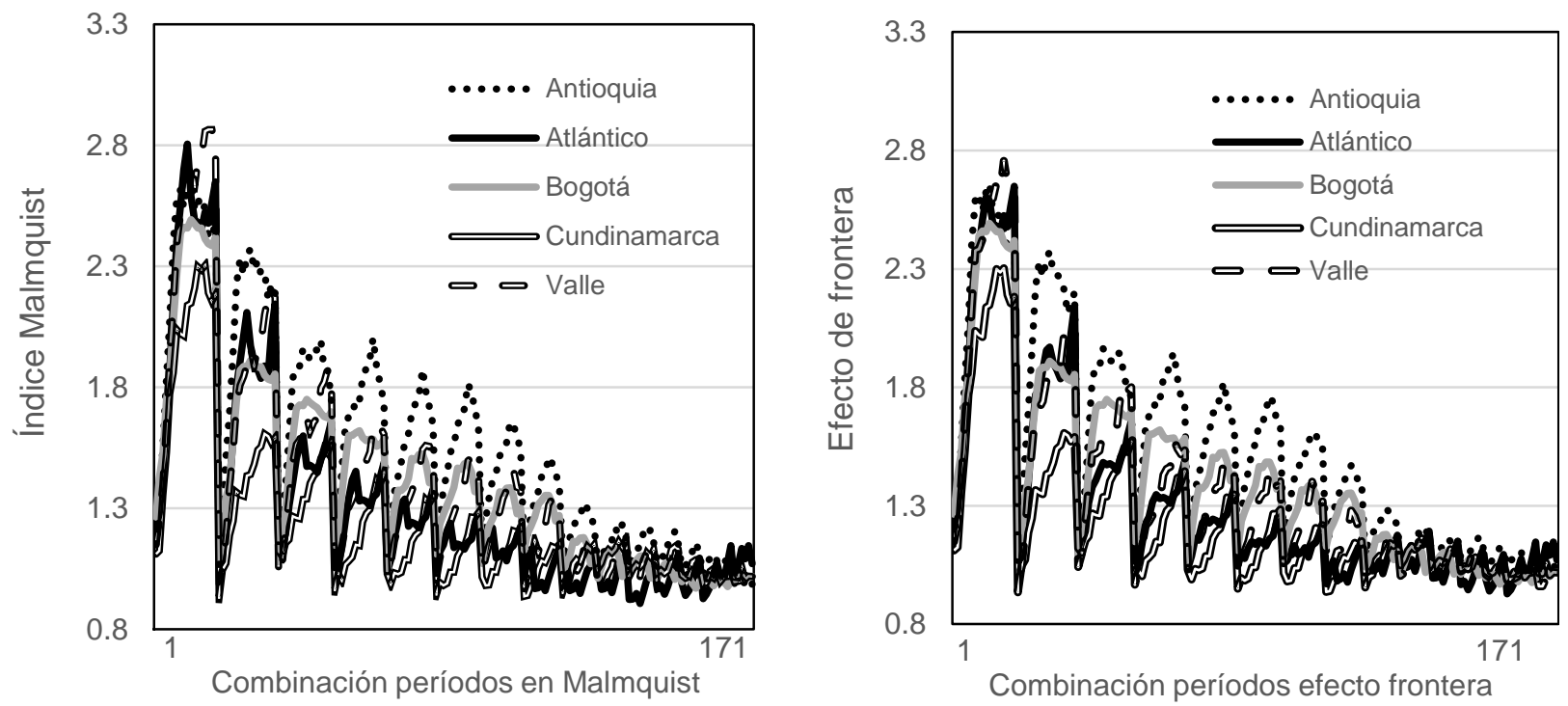

Fig. 3: (a) Comparación Malmquist y (b) efecto de frontera para todas las combinaciones de períodos

Si el efecto de recuperación es mayor a 1 indica progreso en la eficiencia relativa del período 1 al 2 y si efecto de frontera es mayor a 1 indica progreso en la frontera de tecnología alrededor de la UD observada del período 1 a 2 ; si es igual a 1 significa igual estado y, si es menor a 1 indica deterioro en la frontera de tecnología (Cooper et al., 2007).

En la figura 4 se muestra el efecto de recuperación o catch-up como se conoce en el término original. Nótese cómo Atlántico y Valle presentan ciertos deterioros en la frontera de tecnología después de ciertas cambios de períodos, Atlántico después de 8-12, 8-13, . ., 12-13. Aplicaciones del Malquist histórico se pueden dar en la industria y la economía, en general cuando se cuenta con series temporales y se desea monitorear los cambios de eficiencia a través de períodos históricos. Con un programa de software y captura electrónica de datos se pueden calcular Malmquist por lapsos de tiempo y así comparar diferentes unidades con el fin de establecer planes de mejoramiento y apoyar la toma de decisiones, por ello sería un gran soporte a muchos temas de ingeniería.

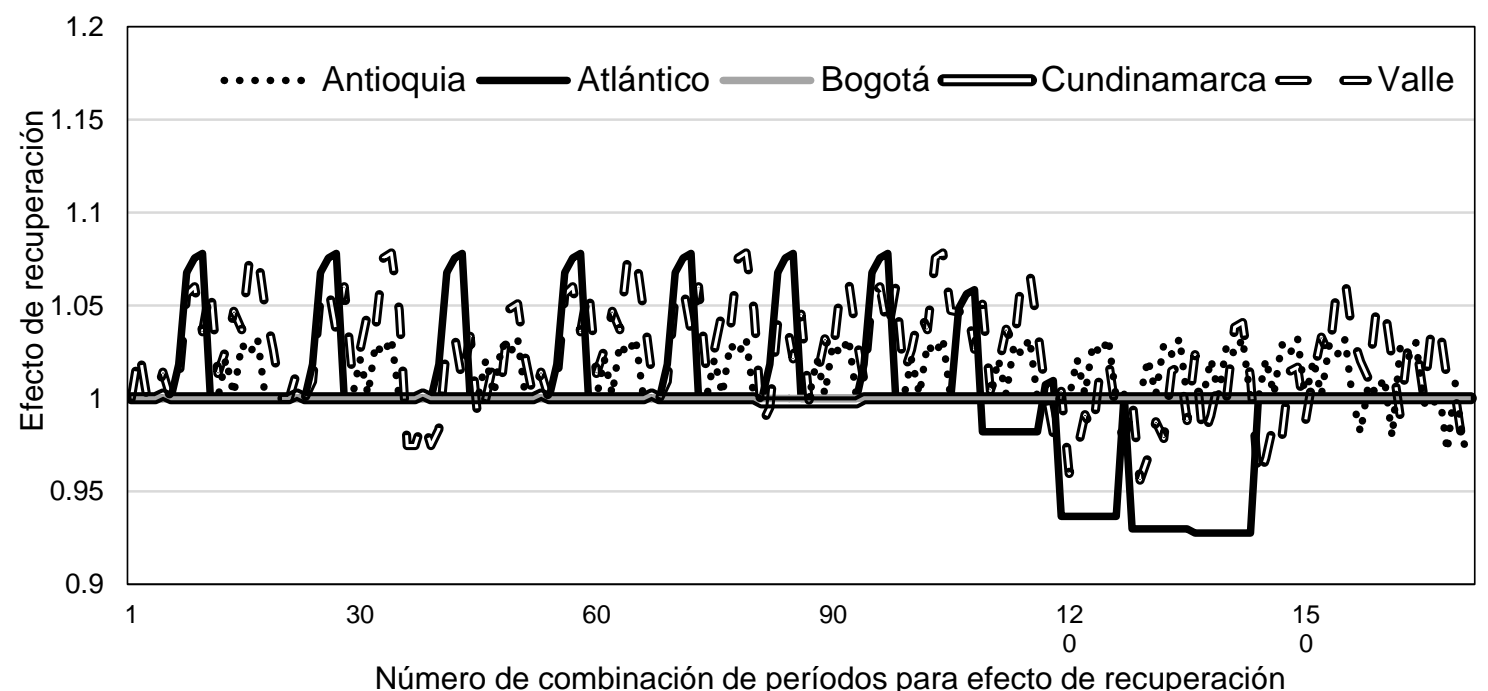

Fig. 4: Efecto de recuperación después de separarlo de los índices Malmquist. 


\section{CONCLUSIONES}

Un nuevo modelo AED temporal (temporal DEA) se ha presentado en el presente artículo ya que tras la exploración de modelos DEA temporales en la literatura se ha encontrado que no se han registrado nuevos métodos a parte del índice Malmquist y el Análisis de ventana. El nuevo AED temporal se presenta como un Malmquist histórico y tiene la ventaja de aceptar series de tiempo como variables de entrada y salida; por lo tanto, hereda las bondades de la técnica de series temporales como poder realizar pronósticos. Una de las grandes ventajas del nuevo modelo es que brinda una información histórica en cuanto a cambio de eficiencias en el tiempo se refiere, lo que constituye una debilidad del Malmquist clásico que sólo evalúa cambios de dos períodos. Se ha mostrado que seleccionar sólo dos períodos de tiempo para calcular cambios de eficiencia, resulta de alguna manera injusto si se desea conocer la eficiencia de las diferentes UDs en su historia. Nuestro nuevo modelo temporal se puede llevar al campo de un AED en tiempo real. Supóngase que por ejemplo, se disponen dispositivos electrónicos que capturen datos de entrada y salida en los procesos productivos durante un lapso de tiempo, nuestro Malmquist histórico tendría la capacidad de calcular no eficiencias instantáneas sino considerar la historia del lapso de tiempo en minutos u horas, dependiendo de la necesidad. Incluso este nuevo índice tiene la capacidad de hacer pronósticos de cómo se comportarían las UDs en los siguientes períodos si se continua con la tendencia histórica, y esto permitiría tomar decisiones en tiempo real.

\section{REFERENCIAS}

Al-Najjar, S. M. y M. A. Al-Jaybajy, Application of Data Envelopment Analysis to Measure the Technical Efficiency of Oil Refineries: A Case Study, doi: 10.5430/ijba.v3n5p64, International Journal of Business Administration, 3(5), 64-77 (2012)

Boqian, L y F. Rilong, Regional differences of $\mathrm{CO}_{2}$ emissions performance in China's agricultural sector: $\mathrm{A}$ Malmquist Index Approach, European Journal of Agronomy, 70, 33-40 (2015)

Chang, Y.T. y otros tres autores, Environmental efficiency analysis of transportation system in China: A nonradial DEA approach, Energy Policy, 58, 277-283 (2013)

Charnes; W.W. Cooper y E. Rhodes, Measuring the Efficiency of Decision Making Units, European Journal of Operational Research, 2, 429-444 (1978)

Chen, Y., Non-radial Malmquist Productivity Index with an Illustrative Application to Chinese Major Industries, International Journal of Production Economics, 83, 27-35 (2003)

Cook, W.W. y L.M. Seiford, Data Envelopment Analysis (DEA) - Thirty years on, European Journal of Operational Research, 192, 1-17 (2009)

Cooper W.W.; L.M. Seiford y K. Tone, Data Envelopment Analysis. A comprehensive text with models, aplications, References and DEA-solver sofware, Segunda edición, 283-340, Springer, N.Y, U.S.A (2007)

Delfín, O.D. y J.C.L Navarro, Productividad total de los factores en las terminales de contenedores en los puertos de México: una medición a través del índice Malmquist, Contaduría y Administración, 60, 663-685 (2015)

Färe, R.; S. Grosskopf. Malmquist indexes and Fisher Ideal Indexes, The Economic Journal, 102, 158-160 (1992)

Herrera F.; A. Mendoza y D. Visbal, Eficiencia en los procesos logísticos en las empresas Certificadas en Basc Medellín Mediante Análisis Envolvente de Datos, Rev.U.D.C.A Act \& Div. Cient, 17(1),265-274 (2014)

López, D. A.; N. Y. García y J. F. Herrera. Desarrollo de un Modelo Predictivo para la Estimación del Comportamiento de Variables en una estructura de Red, Información Tecnológica, 26(5), 143-154 (2015)

Lin, B. y R. Fei, Regional differences of $\mathrm{CO} 2$ emmisions performance in China`s Agricultural Sector: A Malmquist index Aproach, European Journal Agronomy, 70, 33-40 (2015)

Macpherson, A.J.; P.P. Principe y Y.b. Shao, Controlling for exogenous environmental variables when using data envelopment analysis for regional environmental assessments, Journal of Environmental Management, $119,220-229$ (2013)

Mahinda W. y M. Meoli, Productivity chance of microfinance intitutions in Kenya: A bootstrap Malmquist Approach, Journal or Retailing and Consumer Services, 115-121 (2015) 
Maldonado, H. y otros ocho autores, Metodología Encuesta Anual de Manufactura Departamento Nacional de Estadística DANE, 1-108, Bogotá, Colombia (2009)

Meng, F.Y. y otros tres autores, Measuring environmental performance in China's industrial sectors with nonradial DEA, Mathematical and Computer Modelling, 58(5-6), 1047-1056 (2013)

Sala-Garrido, R.; F. Hernández y M. Molinosm, Assessing the efficiency of wastewater treatment plants in an uncertain context: a DEA with tolerances approach, Research Article Environmental Science \& Policy, 18, 34-44 (2012)

Simar, L. y P.W. Wilson, Estimation and inference in two stage, semi parametric models of production processes. Jounal of Econometrics 136, 31-64 (2007)

Sheng, Y. y otros tres autores, Energy trade efficiency and its determinants: A Malmquist index Approach, Energy Economics, 25, 306-314, (2015)

Sueyoshi, T. y S. Aoki. A use of a nonparametric statistic for DEA frontier shift: the Kruskal and Wallis rank test, Omega, 29, 1-18 (2001)

Sueyoshi, T. y M. Goto, Slack adjusted DEA for time series Analysis: performance measurement of Japanese electric power generation industry in 1984-1993, Eur. J. Oper. Research, 133, 232-259 (2001)

Sueyoshi, T. y M. Goto, DEA environmental assessment in a time horizont: Malmquist index on fuel mix electricity and CO2 industrial nations, Energy Economics, 40, 370-382 (2013)

Sueyoshi, T. y M. Goto, DEA Environmental Assesment in Time Horizon: Radial Approach for Malmquist Index Measurement on Petroleum Companies, doi: 10.1016/j.eneco.2015.07.010, Energy Economics (2015)

Tone, K., A Slacks-based Measure of Super-efficiency in Data Envelopment Analysis, European journal of Operational Research, 143, 32-41 (2002)

Toshiyuki, S.; G. Mika y S. Manabu, DEA window analysis for environmental assessment in a dynamic time shift: Performance assessment of U.S. coal-fired power plants, Energy Economics, 40, 845-857 (2013)

Varabyova, Y. y J. Schreyogg, International comparisons of the technical efficiency of the hospital sector: Panel data analysis of OECD countries using parametric and non parametric approaches, Health Policy 112(1-2), 70-79 (2013)

Zhu, J., Data Envelopment Analysis with preference Structure, Journal of the Operational Research Society, 47, 136-150 (1996)

Zhu, J, Quantitaive Models for Performance Evaluation and Benchmarking Data Envelopment Analysis with Spreadsheet, Third Edition 11-19, Springer, Massachusetts, U.S.A (2014) 